\title{
Teste de Bender com disléxicos: comparação de dois sistemas de pontuação
}

\author{
Acácia Aparecida Angeli dos Santos ${ }^{1}$ \\ Lilia Maise de Jorge
}

\begin{abstract}
Resumo
Este estudo teve como objetivo avaliar aspectos maturacionais e disfuncionais referentes à percepção visomotora de disléxicos valendo-se, para isso, do Teste de Bender analisado sob dois sistemas de correção, o sistema de pontuação gradual (B-SPG) e o sistema Lacks. Participaram da pesquisa 20 disléxicos com idade entre 9 e 16 anos $(\mathrm{M}=12)$, sendo 16 do sexo masculino e 4 do sexo feminino. Os dados mostraram que a média de erros dos disléxicos foi acima do que é esperado para crianças de nove e dez anos que compõem a amostra normativa do $B$ SPG. De acordo com o sistema Lacks de pontuação os fatores mais comprometidos na amostra de disléxicos foram referentes a mudanças na forma da gestalt e distorção da gestalt, fatores esses equivalentes à distorção da forma no $B$ SPG. O índice de correlação entre os dois sistemas foi significativo e alto $(r=0,76)$.
\end{abstract}

Palavras-chave: Dislexia; Avaliação; Percepção visomotora.

\section{Bender Test with dyslexics: Comparison of two systems of punctuation}

\begin{abstract}
The present study aimed to evaluate maturity and dysfunctional aspects referred to visualmotor perception of dyslexics using for this, the Bender Test analyzed under two correction systems, being the gradual scoring system (B-SPG) and Lacks system. Twenty dyslexics have taken part in the research with ages from 9 to 16 years (average=12), being 16 males and 4 females. The data showed the average of mistakes committed by the dyslexics was above the expected for children from nine to ten years who compose the B-SPG normative sample. According to the Lacks scoring system the factors more affected in the sample of dyslexics were referred to changes on the gestalt form and distortions, factors which are equivalent to the form distortions on the B-SPG. The correlation index between these two systems was significant $(\mathrm{r}=0.76)$.
\end{abstract}

Keywords: Dyslexia; Evaluation; Visualmotor perception.

\section{Introdução}

A codificação escrita faz parte da história da humanidade há aproximadamente cinco mil anos e passou por várias transformações até chegar ao que hoje está sendo usado no Ocidente como recurso para transmitir e registrar conhecimento, ou seja, o sistema ortográfico alfabético. Analisando descrições sobre a história da escrita, é interessante pensar que o que atualmente se constitui em um conjunto de signos com significado (ou seja, as palavras), não relacionado com sua forma (objeto ou evento), já foi em épocas passadas um desenho similar ao objeto concreto ou imaginário o qual deveria representar (Azcoaga, 1993; Capovilla \& Capovilla, 2000; Santos \& Navas, 2002). A trajetória dos hieróglifos às letras carrega a idéia de que as inscrições não representavam conceitos, mas significados, que emergiam da linguagem da coletividade e não de processos cognitivos.
Palavras, portanto, já foram desenhos e o processo de leitura e escrita tem, em sua origem, um caráter de incorporação do saber coletivo, compartilhado com os outros, que na nossa sociedade atual se tornou uma forma de poder e de diferenciação daqueles que vão ter mais ou menos acesso às oportunidades oferecidas pelo meio. A transposição da relação hieróglifo/objeto ao fonema/grafema trouxe progressos do ponto de vista antropológico, mas trouxe também complicações do ponto de vista social, educacional e psicológico (Ellis, 1995). Segundo Santos e Navas (2002), a escrita é o registro prático e econômico do conhecimento humano, que reflete a capacidade de pensar de forma abstrata sobre sua própria linguagem. Requer habilidades metalingüísticas por parte do aprendiz, que precisa atingir a compreensão do princípio alfabético, para dominar a complexidade do sistema ortográfico.

Falhas na aquisição dessas habilidades,

\footnotetext{
${ }^{1}$ Endereço para correspondência:

Universidade São Francisco - Rua Alexandre Rodrigues Barbosa, 45 - 13251-900 - Itatiba-SP

E-mail: acaciasantos@terra.com.br
} 
provenientes de condições neurobiológicas, fatores hereditários e condições ambientais desfavoráveis, traduzem o que se conhece como dislexia, ou transtorno de leitura. De acordo com a International Dyslexia Association (2005), a dislexia é um distúrbio específico da aprendizagem, caracterizado pela dificuldade de reconhecimento de palavras, de soletração e decodificação. Essas limitações resultam em um déficit fonológico da linguagem que é inesperado em relação a outras habilidades cognitivas e a práticas educativas. Freqüentemente aparecem problemas na compreensão da leitura que podem dificultar a ampliação do vocabulário e a aquisição de conhecimentos.

A dislexia vem sendo estudada desde o início do século passado, e apesar de ser foco de interesse para muitos pesquisadores na atualidade, tem ainda aspectos imprecisos em sua definição, acarretando dificuldades diagnósticas (Snowling, 2004; Van Hout \& Estienne, 2001). Segundo Massi (2004), os indicativos causais desse distúrbio, bem como os sintomas descritos para sua caracterização, carecem de consistência.

Os estudos de Orton, realizados nas décadas de 1920 a 1940, com mais de três mil disléxicos, apontavam para a possibilidade de problemas na linguagem serem os determinantes daquilo que ele denominou estrefossimbolia, ou seja, distorção dos símbolos. Até os anos 70 as hipóteses levantadas para investigação das causas dos distúrbios de leitura e escrita ainda permaneceram pautadas na deficiência do processamento visual (Van Hout \& Estienne, 2001). Instrumentos de avaliação como o Teste Gestáltico Visomotor de Bender, Cubos de Kohs, Figuras Complexas de Rey (1998/1999) e similares, que investigavam a percepção visual e a organização visoespacial, têm sido usados para o diagnóstico da dislexia há muitos anos (Capovilla \& Capovilla, 2000).

Nessa linha de investigações, Lopez (1995) considera que o desenvolvimento de aspectos da motilidade ocular como convergência, acomodação, direção e orientação espacial afetam a qualidade da leitura e da escrita em crianças na fase escolar. Santos, Behlau e Caovilla (1995) avaliaram os movimentos de regressão e de fixação em tarefa de leitura de texto, a partir de nistagmografia computadorizada. Embora não tenha sido evidenciada a dificuldade em fixação ocular, as crianças com distúrbio de leitura apresentaram maior número de regressões oculares do que as do grupo controle. Valendo-se de delineamento de pesquisa similar, Problano, Caballero, Castilho e Cortés (1996) investigaram, por meio de eletrooculografia, crianças com distúrbio de aprendizagem.
Essas crianças mostraram um tempo de leitura maior e um número maior de movimentos sacádicos e regressivos em comparação às do grupo-controle.

Santos e Navas (2002) assinalam que em estudos mais recentes sobre o tema os movimentos de regressão ocular são maiores em crianças com distúrbio de leitura e aumentam de acordo com a dificuldade da tarefa, podendo tornar a leitura ineficiente. Taylor (2004) comenta sobre as dificuldades perceptuais visuais e visomotoras que algumas crianças experimentam, salientando que esses problemas podem acarretar dificuldades na lembrança das formas das letras, da seqüência dos movimentos requeridos para traçar as letras e da ordenação das letras na palavra. Podem ocasionar ainda inversões, desajustes nos tamanhos das letras e dos espaços entre as palavras, em especial em crianças com dislexia que exercitam menos a escrita por acharem trabalhosa a tarefa de registro gráfico.

No entanto, não há consenso nas explicações sobre os fatores preponderantes associados à dislexia. Essa controvérsia é ilustrada por Van Hout e Estienne (2001), que descrevem os estudos de Vellutino descartando a possibilidade causal de problemas visoespaciais para a dislexia, enfatizando que os déficits para este distúrbio seriam de ordem verbal ou fonológica. Relatam ainda estudos nos quais alguns disléxicos teriam desempenhos superiores para a orientação espacial e para a memória topográfica. Mediante a falta de consistência dos dados para confirmar a hipótese causal de deficiência na percepção visual em disléxicos, os estudos se encaminharam para a pesquisa de déficits verbais e não mais visuais.

Adotando uma nova perspectiva, algumas pesquisas têm sido direcionadas a investigações do processamento de informações auditivas, memórias de trabalho e de longo prazo, velocidade de processamento, investigações essas que permitem considerar que os déficits fonológicos e metafonológicos conduzem a dificuldades de leitura e escrita (Capovilla, Gütschow \& Capovilla, 2004; Salles \& Parente, 2002).

Nessa mesma direção são apresentados resultados de estudos feitos com imagens do funcionamento cerebral, indicando que o problema principal da dislexia é fonológico. Assim, demonstram que são observadas alterações do processamento neurológico central que podem ser detectadas em testes específicos de processamento auditivo (Sauer e cols., 2006; Shaywitz, 2006).

O estudo longitudinal desenvolvido desde 1983 por Shaywitz (2006), em Connecticut nos Estados Unidos, tem permitido a constatação de 
alguns aspectos importantes em dislexia, como o fato de não existir um ponto de corte separatista entre os bons e os maus leitores. A autora defende a idéia de que a dislexia ocorre num continum, sendo mais bem explicada por um modelo dimensional e não por um modelo categórico, cujo ponto de corte seja arbitrário. Esse estudo também traz dados sobre a prevalência da dislexia, apontando uma proporção de 1:5 nas crianças norte-americanas, sendo três ou quatro vezes mais presente em meninos do que em meninas.

Das dificuldades apresentadas por disléxicos em idade escolar, descritas na literatura brasileira atual, destacam-se a inabilidade para a aprendizagem da leitura e, conseqüentemente, para a expressão escrita; dificuldades na fixação do alfabeto, de tabuadas e de seqüências de eventos; falta de atenção e de concentração; dificuldade em memória de curto prazo; dificuldades com rima e aliteração. São também referidas inabilidades psicomotoras e desorganização geral; confusão com orientações de direita e esquerda; trocas nas ordenações de números e palavras; além de problemas emocionais advindos de um histórico de frustração em relação ao desempenho escolar (Capovilla \& Capovilla, 2002; Mico \& Barreiro, 2004, Schirmer, Fontoura \& Nunes, 2004)

Sendo a dislexia um tema complexo, torna-se um campo vasto para pesquisas de toda ordem, tanto em relação ao aprofundamento do conhecimento acerca dos aspectos relacionados ao processamento fonológico, quanto à investigação de outros aspectos ainda pouco explorados que acompanham os distúrbios de leitura e escrita. Estudiosos da área ressaltam a importância de ampliação das pesquisas que abordem as relações entre leitura e escrita e outras habilidades cognitivas, incluindo as visoespaciais (Capovilla, Gütschow \& Capovilla, 2004).

Ao lado disso, de acordo com Snowling (2004), o fato de não haver evidência causal dos fatores visuais sobre a dislexia não impede que dificuldades no processamento perceptual visual e déficits na memória visual possam exacerbar os problemas manifestados pelos disléxicos. A autora atenta para o fato de que alguns disléxicos apresentam dificuldades conjuntas em caligrafia, e cita estudos sobre o controle motor ocular, demonstrando que crianças com dislexia apresentam menor índice que as leitoras normais em definição da preferência ocular, o que traz complicações na visão binocular.

A importância de se investigar as múltiplas habilidades que podem estar comprometidas em disléxicos tem levado à escolha do Teste Gestáltico Visomotor de Bender como instrumento de avaliação do aspecto percepto-motor. Sua nova versão Psico-USF, v. 12, n. 1, p. 13-21, jan./jun. 2007 americana - Bender Gestalt II (Brannigan \& Decker, 2003) - conta com estudos de validade que foram realizados com população clínica, entre os quais o de distúrbios de leitura, com 185 indivíduos, de ambos os sexos, em idades de 6 anos e meio a 19 anos e meio. Os desempenhos dos disléxicos foram inferiores ao grupo de referência, tanto na tarefa de cópia quanto na de memória.

Num estudo de avaliação interdisciplinar da dislexia de desenvolvimento, proposto por Pestun, Ciasca e Gonçalves (2002), o Bender também aparece como instrumento utilizado na bateria de testes selecionados pela equipe, em sua versão de correção pelo método de Clawson. Esse estudo se propôs a avaliar um caso de criança disléxica, reunindo informações médicas, neuropsicológicas e pedagógicas. No caso, o escore do Bender apresentou-se com nível maturacional abaixo do esperado para a idade cronológica da criança estudada. Os dados obtidos permitiram concluir que o caso em questão apresentava a chamada dislexia mista, com comprometimentos tanto no processamento fonológico quanto no processamento visual envolvidos no ato de ler.

O Teste de Bender, em suas versões mais comuns de correção (Koppitz, 1989; Clawson, 1982) foi usado clinicamente no Brasil até recentemente, quando nos anos de 2001 e 2003 o Conselho Federal de Psicologia (CFP) baixou resoluções relacionadas à utilização de instrumentos de avaliação psicológica, requerendo estudos de validação e precisão para a realidade brasileira. Reconhecendo que este teste tem um histórico que lhe garante um lugar de destaque em avaliação psicológica de crianças, Sisto, Noronha e Santos (2005) realizaram um estudo com o Bender, propondo um sistema de pontuação gradual para o critério específico de distorção da forma. Seu uso está previsto para crianças de idades entre 6 e 10 anos, podendo ser aplicado coletiva ou individualmente.

Com o uso de aplicação coletiva desse sistema, Suehiro e Santos (2005) se propuseram a investigar o desempenho de 287 alunos de $2^{\mathrm{a}}$ s. e $3^{\mathrm{a}} \mathrm{s}$. séries do ensino fundamental (atualmente $3^{\circ}$ e $4^{\circ}$ ano do ensino básico). Os resultados obtidos indicaram a existência de diferenças significativas no desempenho de crianças diagnosticadas como portadoras de dificuldades de aprendizagem, classificadas nas categorias previstas na Escala de Avaliação de Dificuldades na Aprendizagem da Escrita (ADAPE), de Sisto (2001). Assim, crianças com dificuldades de aprendizagem leve, média e acentuada apresentaram mais erros de distorção de forma do que as sem indícios de dificuldades de aprendizagem, tendo alcançado a média de erros de 8,20 no Bender-Sistema de Pontuação Gradual. As 
autoras recomendam que outras pesquisas sejam realizadas, visando à ampliação do conhecimento sobre a sensibilidade do sistema para avaliação dessa população.

Com base em pesquisas feitas com profissionais que atuam em neuropsicologia nos Estados Unidos, Lacks (1999) afirma que o Bender é um dos testes mais utilizados na prática neuropsicológica (o quarto mais usado), visto ser a habilidade percepto-motora uma das funções cognitivas. Em seu trabalho de revisão do uso do Bender, Lacks (1999) propõe um sistema de avaliação para ser usado como um screening, ou seja, como uma investigação inicial de disfunção cerebral.

$\mathrm{Na}$ adaptação feita por Lacks (1999), os doze fatores discriminativos de danos cerebrais fazem parte da lista proposta por Hutt e Briskin em 1960, que se agrupavam originalmente em cinco categorias mais amplas, ou seja, organização (incluindo colisão), tamanho (incluindo coesão), mudanças na forma da gestalt (incluindo dificuldade em fechamento e em angulação), distorção da gestalt (incluindo rotação, regressão, simplificação, fragmentação, dificuldade em sobreposição e perseveração), movimento e desenho (incluindo falta de coordenação motora). O erro denominado impotência não estava incluído nas cinco categorias. Utilizando esse novo sistema, a autora descreve estudos com a população focalizada por ela, especialmente com pacientes adultos psiquiátricos, adultos sem história clínica de doença mental e, também, com pessoas idosas.

Estudos com adolescentes de 12 a 16 anos, valendo-se de comparação entre as pontuações dos sistemas Lacks e Koppitz, foram efetuados por McIntosh e cols., em 1988, e são citados por Lacks (1999), mostrando um índice de correlação significativa entre ambos $(r=0,71)$. Os indicadores de consistência interna de ambos os sistemas foram considerados excelentes (Koppitz com $r=0,97$ e Lacks com $r=0,93$ ). Segundo Lacks, a adolescência é uma fase para a qual ainda não havia estudos de validade para o Bender. Os estudos de Koppitz comportavam até a idade de 11 anos e os de Lacks começavam com 18 anos. As contribuições de McIntosh foram, portanto, não apenas de alimentar com dados esta faixa etária, mas de trazer à reflexão se o desenvolvimento percepto-motor teria seu platô após os 11 anos de idade e se o sistema Lacks seria sensível à detecção de sinais de disfunção cerebral também nesta fase de desenvolvimento. $\mathrm{O}$ decréscimo de erros com o aumento da idade não ficou evidenciado nesse estudo.

Partindo do pressuposto que na dislexia há aspectos maturacionais disfuncionais referentes à percepção visoespacial, a presente pesquisa foi proposta para comparar os resultados obtidos por crianças e adolescentes que possuem diagnóstico de Transtorno da Leitura, comparando-se os resultados no Teste de Bender, de acordo com os sistemas B-SPG e com Lacks, correlacionando-se os dois sistemas de pontuação. Análises da freqüência de pontuações nas várias figuras nos dois sistemas de pontuação, bem como das comparações entre as médias do grupo normativo do BSPG e do grupo de disléxicos foram focos de discussão nesta pesquisa.

\section{Método}

\section{Participantes}

A amostra foi composta por 20 portadores de dislexia do desenvolvimento, sendo 16 do sexo masculino $(80 \%)$ e 4 do sexo feminino (20\%), na faixa etária entre 9 e 16 anos (Média $=12$ e DP $=2,08$ ). Os participantes tiveram seus diagnósticos realizados por equipe especializada $(\mathrm{N}=8)$ ou por pelo menos dois profissionais da área da saúde $(\mathrm{N}=12)$, tomando-se por base os critérios propostos no DSM-IV-TR (2003). Por se tratar de uma população eminentemente clínica, tais participantes foram provenientes de consultórios particulares de psicólogos, fonoaudiólogos e médicos, de instituições que prestam atendimento clínico, e de escolas municipais da região do Vale do Paraíba, estado de São Paulo, durante o ano de 2005. O nível de escolaridade variou de $3^{\mathrm{a}}$ a $7^{\mathrm{a}}$ série do ensino fundamental (atualmente $4^{\circ}$ a $8^{\circ}$ ano do ensino básico).

\section{Instrumento}

O instrumento utilizado neste estudo foi o Teste Gestáltico Visomotor de Bender corrigido mediante dois sistemas. Um deles é o Sistema de Pontuação Gradual (B-SPG), de Sisto, Noronha e Santos (2005) em sua forma de aplicação individual, com uso de cartões. O segundo é o Sistema de Pontuação de Lacks (1999), também com aplicação individual dos nove cartões.

O B-SPG se propõe a avaliar a maturação percepto-motora de crianças com idade entre 6 e 10 anos, mediante análise da distorção da forma nas nove figuras originais de Bender. As pontuações variam de 0 a 2 para cada figura e, somente para a figura 6 , têm variação de 0 a 3 pontos, com a possibilidade de um escore total de até 21 pontos. Tal proposta de correção do teste, utilizando-se de pontuação gradual do erro, teve seus estudos apoiados numa amostra de 1.052 crianças, de pré-escola a $4^{\mathrm{a}}$ série do ensino fundamental (correspondentes ao $1^{\circ}$ a $5^{\circ}$ anos atuais do ensino básico), freqüentando escolas públicas, municipais e estaduais, de cidades do interior de São Paulo. Estudos de correlação entre idade e pontuação $(r=-0,58)$ por um lado, e idade e diferenças de médias dos grupos etários $[\mathrm{F}(4,1048)=155,15]$, por outro, mostram que o 
teste é sensível em relação a mudanças desenvolvimentais. Análises de precisão do teste, para essa versão, acusam boa consistência interna (alfa de Cronbach 0,80; SpearmanBrown 0,77 e modelo Rasch 0,76).

O sistema de pontuação do Teste de Bender desenvolvido por Lacks consiste numa adaptação do Sistema de Hutt-Briskin, de 1960, utilizado como screening para detecção de disfunções cerebrais. A revisão mais atualizada dos estudos de Lacks (1999) oferece dados acerca da precisão e da validade desse sistema para uso clínico, além de conter critérios novos para interpretação dos resultados em grupos diferenciados de adolescentes e idosos.

Os doze fatores analisados são referentes à rotação, dificuldade em sobreposição de figuras, simplificação, fragmentação, regressão, perseveração, colisão, impotência, dificuldade em fechamento, falta de coordenação motora, dificuldade em angulação e coesão. As pontuações finais podem variar de 0 a 12 , numa escala na qual 0 a 3 pontos significa ausência de dano cerebral, 4 pontos significa situação borderline, 5 e 6 pontos significa alguma evidência de dano, 7 e 8 pontos sugere forte evidência de dano, e 9 a 12 pontos sugere muito forte evidência de dano ou deterioração cerebral.

Esses dois sistemas de pontuação foram escolhidos por terem seus enfoques de avaliação diferenciados, ou seja, maturação percepto-motora e disfunção cerebral, porém voltados para aspectos de interesse na investigação da dislexia. A Tabela 1 resume as características principais de cada sistema.

Tabela 1 - Especificidade dos sistemas de avaliação

\begin{tabular}{|c|c|c|}
\hline Características & B-SPG & Lacks \\
\hline Objetivo & Avaliar maturação percepto-motora & $\begin{array}{l}\text { Screening para detecção de disfunções } \\
\text { celebrais }\end{array}$ \\
\hline Aplicação & Coletiva ou individual & Individual \\
\hline Critérios & $\begin{array}{l}\text { Avalia distorção da forma } \\
\text { Uso clínico e educacional }\end{array}$ & $\begin{array}{l}\text { Avalia } 12 \text { fatores de disfunção } \\
\text { cerebral } \\
\text { Uso clínico }\end{array}$ \\
\hline Pontuação & $\begin{array}{l}\text { Máxima de } 21 \text { pontos } \\
0 \text { a } 3 \text { pontos para figura } 6 \\
0 \text { a } 2 \text { pontos para demais figuras }\end{array}$ & $\begin{array}{l}\text { Máximo de } 12 \text { pontos } \\
1 \text { ponto para cada fator }\end{array}$ \\
\hline Idade & $\begin{array}{l}\text { Pontua-se a intensidade da deformação } \\
6 \text { a } 10 \text { anos }\end{array}$ & $\begin{array}{l}\text { Pontua-se o desvio } \\
12 \text { a } 65 \text { anos e }+\end{array}$ \\
\hline
\end{tabular}

\section{Procedimento}

O estudo foi aprovado previamente pelo Comitê de Ética da Universidade São Francisco, e todos os responsáveis legais pelos participantes assinaram o Termo de Consentimento Livre e Esclarecido, permitindo que seus filhos participassem da pesquisa. Dados referentes ao diagnóstico foram colhidos com os pais ou responsáveis antes da avaliação.

A aplicação ocorreu de forma individual, em consultórios, sem estímulos gráficos visuais no ambiente próximo. Após um rapport, foi colocada à frente do examinando uma folha em branco, juntamente com um lápis, e os cartões foram apresentados, um a um, para que fossem observados e copiados. Observações gerais acerca de seu desempenho foram anotadas pela examinadora numa ficha de anotações.

\section{Resultados}

Os dados foram analisados primeiramente considerando os critérios propostos no B-SPG, definindo-se as porcentagens de pontuação do grupo de disléxicos em cada figura do teste, bem como cada grau de intensidade da distorção da forma Os resultados obtidos estão apresentados na Tabela 2.

Tabela 2 - Freqüências de pontuação com porcentagens para cada figura no B-SPG

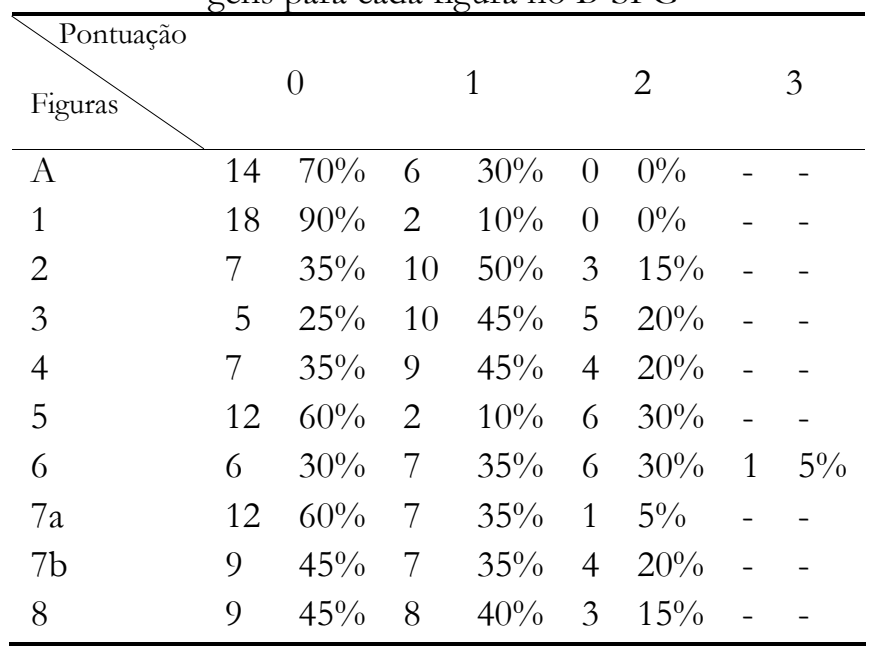


Pela análise das pontuações, verificou-se que nas figuras A, 1, 5 e 7a (hexágono vertical) as crianças tiveram porcentagens de acerto igual ou acima de $60 \%$, ou seja, as porcentagens de erro dessas figuras foram menores. Já nas figuras 2, 3, 4 e 6 tiveram porcentagens de erro igual ou acima de $65 \%$, considerando-se dois níveis de pontuação para as figuras 2,3 e 4, e três níveis para a figura 6 . As figuras $7 \mathrm{~b}$ (hexágono inclinado) e 8 tiveram porcentagens de erro em 55\% dos casos, considerando-se também dois níveis de pontuação.

Julgou-se importante comparar a pontuação encontrada nos disléxicos, tanto os que estavam dentro quanto os que estavam acima da faixa etária prevista na amostra, com a média estimada para o grupo normativo do B-SPG. Esses dados encontram-se demonstrados na Figura 1.

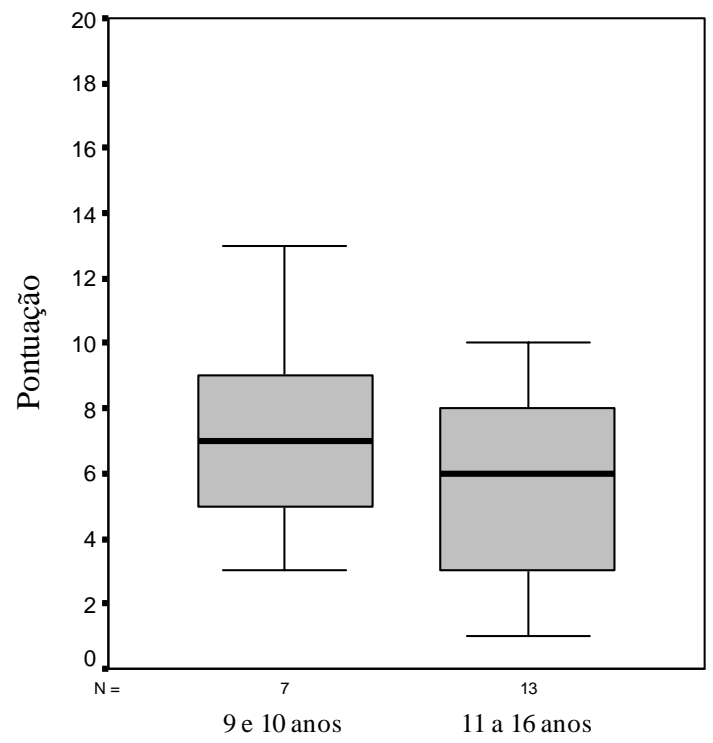

Figura 1 - Comparação das pontuações do grupo de disléxicos com a amostra normativa

Os resultados obtidos permitem a constatação de que a performance do grupo clínico na cópia das figuras, considerando-se as faixas etárias de 9 e 10 anos e de 11 a 16 anos, é inferior à da amostra normativa. No grupo acima de 10 anos a pontuação da idade foi comparada com a pontuação média da idade limite de investigação deste sistema, que é de 5,79 para meninos de 10 anos e 4,99 para meninas.

Investigando-se agora os dados, de acordo com o Sistema de Lacks, observa-se que os índices de maior freqüência de erros encontram-se nos fatores Dificuldade em Fechamento (55\%), Falta de Coordenação Motora $(40 \%)$ e Dificuldade em Angulação (40\%), conforme demonstrado na Tabela 3.
O fator Coesão não foi pontuado e a Colisão foi manifestada por apenas um dos sujeitos. De um modo geral as incidências nos fatores não foram altas.

Tabela 3 - Freqüência e percentual de erros pontuados para cada fator do Sistema Lacks

\begin{tabular}{lcc}
\hline \multicolumn{1}{c}{ Fatores } & $\begin{array}{c}\text { Freqüência } \\
\text { de erros }\end{array}$ & Porcentagem \\
\hline Rotação & 4 & $20 \%$ \\
Dificuldade em & 5 & $25 \%$ \\
sobreposição & 5 & $25 \%$ \\
Simplificação & 3 & $15 \%$ \\
Fragmentação & 4 & $20 \%$ \\
Regressão & 6 & $30 \%$ \\
Perseveração & 1 & $5 \%$ \\
Colisão & 3 & $15 \%$ \\
Impotência & 11 & $55 \%$ \\
$\begin{array}{l}\text { Dificuldade em } \\
\text { fechamento }\end{array}$ & & \\
$\begin{array}{l}\text { Falta de } \\
\text { coordenação }\end{array}$ & 8 & $40 \%$ \\
motora & & $40 \%$ \\
Dificuldade em & 8 & $0 \%$ \\
angulação & 0 & \\
Coesão & &
\end{tabular}

Os dois sistemas de pontuação, B-SPG e Lacks, foram analisados mediante o estabelecimento do coeficiente de correlação de Spearman para verificação do índice de associação entre eles. O resultado foi significativo, acusando alta correlação $\left(r_{o}=0,76\right)$, com nível de significância fixado em $p \leq 0,01$, sugerindo que ambos os sistemas estão medindo aspectos semelhantes (ou congruentes) no que se refere à avaliação de disléxicos.

\section{Discussão}

Os dados analisados neste estudo mostraram que o índice total de erros encontrados no grupo de disléxicos foi acima do que é esperado para crianças de nove e dez anos que apresentam condições normais de aprendizagem, de acordo com dados contidos do manual do B-SPG (Sisto, Noronha \& Santos, 2005). Isso indica haver comprometimento da habilidade percepto-motora de crianças e adolescentes diagnosticados como disléxicos, sendo importante mais uma vez salientar que, nessa análise, a idade dos sujeitos avaliados era, em média, superior à população que compõe os dados normativos do teste, e que serviram de base para a comparação efetuada.

Tais dados também sugerem que, apesar dos vários estudos que indicam o déficit fonológico como o 
fator mais freqüentemente associado à dislexia (Capovilla \& Capovilla, 2002; Massi, 2004; Salles \& Parente, 2002; Shaywitz, 2006), outros fatores também podem comprometer o desempenho em leitura e escrita de disléxicos e contribuir para uma maior compreensão e caracterização do quadro (Lopez, 1995; Snowling, 2004).

Os resultados coletados na amostra de disléxicos aqui estudados são compatíveis com os do estudo referente ao Bender Gestalt II (Brannigan, 2003), efetuado com 185 indivíduos, no qual o desempenho do grupo clínico por ele estudado também se apresentou inferior em relação ao grupo normativo. No mesmo sentido, é congruente com os resultados obtidos por Suehiro e Santos (2005), que também obtiveram, em crianças portadoras de dificuldades de aprendizagem, média maior de erros do que os que a amostra normativa indica para a faixa etária em questão. É importante destacar, ainda, que a média obtida pelas autoras é mais elevada do que a obtida entre os integrantes da amostra clínica focalizada no presente estudo. No entanto, há diferenças entre a forma de aplicação, visto que a apresentação dos cartões individualmente e num ambiente especial (consultório) pode ter favorecido o melhor desempenho do que o conseguido por crianças com dificuldades de aprendizagem em sala de aula. Este aspecto merece ser mais bem explorado em pesquisas futuras, visto que o Sistema B-SPG está proposto para aplicações coletivas ou individuais.

Verificando-se as porcentagens de erro apresentadas pelos disléxicos em cada figura pontuada pelo B-SPG, tanto na investigação do grupo como um todo quanto diferenciados por idade, pode-se considerar que as figuras 2 e 3 , que apresentaram índices relativamente altos, devam ser configurações complexas que dificultem a percepção da gestalt por serem formadas por conjuntos de pequenos elementos (círculos e pontos, respectivamente) distribuídos em seqüências regulares. A figura 5, apesar de ser constituída por pontos, oferece uma gestalt mais bem definida, e quando há pontuação de erro, a porcentagem maior se concentra no tipo 2, ou seja, distorção mais acentuada da forma. Esses dados parecem estar de acordo com os estudos acerca dos movimentos sacádicos em disléxicos, que se apresentam comprometidos sobretudo em movimentos de regressão ocular, dificultando a leitura de textos (Santos \& Navas, 2002).

Com relação à figura 7 , composta por dois hexágonos (7a e 7b), pôde-se observar que o elemento disposto na vertical teve menor índice de erros que o inclinado. Seguindo o mesmo raciocínio, as figuras $4 \mathrm{e}$ 6, que contêm elementos dispostos na diagonal, Psico-USF, v. 12, n. 1, p. 13-21, jan./jun. 2007 também tiveram índices altos na porcentagem de erros, sugerindo que esse detalhe da orientação espacial gráfica possa estar representando uma das dificuldades perceptivas presentes em disléxicos, que deve ser mais bem investigada por meio de outros instrumentos, como o Teste das Figuras Complexas de Rey (Rey, 1998/1999), por exemplo.

Em relação ao uso clínico do Bender como screening para danos cerebrais, segundo o Sistema Lacks de pontuação, as análises efetuadas puderam oferecer apenas índices de porcentagem de erro em virtude do fato de esse sistema não ter normas brasileiras com as quais pudessem ser feitas comparações, nem normas para população com faixa etária menor do que 12 anos. Os dados coletados nesta pesquisa podem servir como ponto de partida para outras, efetuadas com crianças e/ou com diversos grupos clínicos, valendo-se desse sistema de pontuação.

Os resultados referentes às porcentagens encontradas em cada um dos doze fatores previstos nesse sistema permitiram, no entanto, que fosse feita uma investigação mais minuciosa sobre sua classificação. Assim, os fatores mais pontuados na amostra de disléxicos deste estudo foram referentes a mudanças na forma da gestalt, seguidos de distorção da gestalt.

Os itens relacionados à mudança e distorção da gestalt, do sistema Hutt-Briskin, adaptado por Lacks, se assemelham ao que é considerado distorção da forma no B-SPG, fato esse provavelmente responsável pelo índice de correlação significativo encontrado entre as duas pontuações $(r=0,76)$. Tal dado tem particular importância para o B-SPG, sistema que possui normas brasileiras, tendo em vista ampliar sua possibilidade de análise para além da maturação percepto-motora.

\section{Considerações finais}

Os resultados obtidos permitem identificar o Bender-Sistema de Pontuação Gradual como uma proposta de correção que aceita que sejam consideradas nuanças no comprometimento perceptivo-motor, ao invés de se considerar apenas a presença ou ausência de determinado fator na produção do desenho do sujeito. Nesse sentido, proporciona maior flexibilidade nas interpretações dos dados, fator esse de suma importância em contexto clínico, sobretudo para a elaboração de propostas de intervenção.

Vale ressaltar que os indícios percebidos nas cópias dos disléxicos avaliados pelos dois sistemas (BSPG e Lacks) devem ser confirmados mediante aplicação de outros instrumentos, combinados com a experiência clínica. Segundo Lacks (1999), o resultado 
isolado do Bender não fornece dados suficientes para compreensão de quadros clínicos, mas é útil como medida de função específica em meio a um conjunto de testes.

As discussões decorrentes da análise dos dados coletados neste estudo não pretenderam ser conclusivas, principalmente porque a amostra estudada era composta por poucos sujeitos. No entanto, comporta a sugestão de que outras pesquisas devam explorar mais os vários aspectos do desenvolvimento que podem também afetar a performance escolar ou, conforme explica Snowling (2004), possam exacerbar os problemas enfrentados por crianças e adolescentes que apresentam dificuldades na aprendizagem.

Finalmente pode-se, com base nos achados deste estudo, propor que o B-SPG possa ser estudado com populações de idade acima de 10 anos. Seria importante que se investigasse a aplicação coletiva e individual em grupos com diferentes níveis de comprometimentos no desenvolvimento, especialmente porque a gradação da pontuação que o sistema apresenta pode torná-lo mais sensível às diferenças individuais.

\section{Referências}

APA (2003). Manual diagnóstico e estatístico de transtornos mentais DSM-IV-TR. Tradução: Cláudia Dornelles. $4^{a}$ ed. revisada. Porto Alegre: Artes Médicas.

Azcoaga, J. E. (1993). Aspectos neupsicológicos del aprendizaje de la lectura y la escritura. Em N. Rodrigues \& L. L. Mansur. Temas em neuropsicologia (pp. 79-97). São Paulo: Tec Art.

Brannigan, G. G. \& Decker, S. L. (2003). Bender Visual Motor Gestalt Test: Examiner's manual. Riverside Publishing.

Capovilla, A. G. S. \& Capovilla, F. C. (2000). Problemas de leitura e escrita. São Paulo: Memnon.

Capovilla, A. G. S. \& Capovilla, F. C. (2002). Etiologia, avaliação e intervenção em dislexia do desenvolvimento. Em F. C. Capovilla (Org.) Neuropsicologia e aprendizagem: uma abordagem multidisciplinar (pp. 49-75). São Paulo: SBNp; Scor Tecci.

Capovilla, A. G. S., Gütschow, C. R. D. \& Capovilla, F. C. (2004). Habilidades cognitivas que predizem competência de leitura e escrita. Psicologia: Teoria $e$ Prática, 6 (2), 13-26.

Clawson, A. (1982). Bender infantil: manual de diagnóstico clínico. $2^{\mathrm{a}}$ edição. Porto Alegre: Artes Médicas.
Conselho Federal de Psicologia (2003). Resolução ${ }^{\circ}$ 002/2003. Disponível em: < http://www.pol.org.br/ legislacao/doc/resolucao2003_2.doc $>$. Acesso em: maio 2006.

Ellis, A. W. (1995). Leitura, escrita e dislexia: uma análise cognitiva. Porto Alegre: Artes Médicas.

International Dyslexia Association (IDA). What is dyslexia? Disponível em : <http://www.interdys.org/ servlet $/$ compose?section_id=5\&page_id=95\#What $\% 20$ is \%20dyslexia?>. Acesso em: out. 2005.

Koppitz, E. M. (1989). O Teste Gestáltico Bender para crianças. Porto Alegre: Artes Médicas.

Lacks, P. (1999). Bender Gestalt screening for brain dysfunction. $2^{\mathrm{a}}$ edição. New York: John Wiley \& Sons, Inc.

Lopez, T. C. S. (1995). Alterações óculo-motoras e alterações na aprendizagem da leitura e da escrita. Pró-fono, 7(1), 6-8.

Massi, G. A. (2004). Dislexia ou processo de aquisição da escrita? Distúrbios da Comunicação, 16(3), 355-369.

Mico, M. A. \& Barreiro, M. M. (2004). Dislexia. Em L. E. L. $\mathrm{R}$ Vale. (Org.). Temas multidisciplinares de neurologia e aprendizagem (pp. 475-486). São Paulo: Robe.

Pestun, M. S. V., Ciasca, S. \& Gonçalves, V. M. G. (2002). A importância da equipe interdisciplinar no diagnóstico de dislexia do desenvolvimento. Arquivos de Neuro-Psiquiatria, 60(2A), p. 328-332.

Problano, A., Caballero, B. C., Castillo, I. \& Cortés, V. (1996). Electro-oculografic recordings reveal reading deficiences in learning disabled children. Arquives Medical Research, 27(4) 509-512.

Rey, A. (1988/1999). Figuras Complexas de Rey. São Paulo: Casa do Psicólogo.

Salles, J. F. \& Parente, M. A. M. P. (2002). Processos cognitivos na leitura de palavras em crianças: relações com compreensão e tempo de leitura. Psicologia: Reflexão e Crítica, 15(2), 321-331.

Santos, M. T. M. \& Navas, A. L. G. P. (2002). Distúrbios de leitura e escrita: teoria e prática. São Paulo: Manole.

Santos, M. T. M., Behlau, M. S. \& Caovilla, H. H. (1995). Crianças com distúrbio de leitura e escrita: movimentos oculares na leitura à nistagmografia computadorizada. Revista Brasileira de Medicina e Otorringolaringologia, 2(2), 100, 103-104, 106-107.

Sauer, L., Pereira, L. D., Ciasca, S. M., Pestun, M., \& Guerreiro, M. M. (2006). Processamento auditivo e 
SPECT em crianças com dislexia. Arquivos de Neuropsiquiatria, 64(1),108-111.

Schaywtiz, S. (2006). Entendendo a dislexia: um novo e completo programa para todos os niveis de problemas de leitura. Porto Alegre: Artmed.

Schirmer, C. R., Fontoura, D. R. \& Nunes M. L. (2004). Distúrbios da aquisição da linguagem e da aprendizagem. Jornal de Pediatria, 80(2), 95-103.

Sisto, F. F. (2001). Dificuldade de aprendizagem em escrita: um instrumento de avaliação. Em F. F. Sisto, E. Boruchovitch, L. D. T. Fini, R. P. Brenelli \& S. C. Matinelli (Orgs.). Dificuldades de aprendizagem no contexto psicopedagógico (pp. 190-213). Petrópolis, RJ: Vozes.

Sisto, F. S., Noronha, A. P. P. \& Santos, A. A. A. (2005). Teste Gestáltico Visomotor de Bender: Sistema de Pontuação Gradual (B-SPG) - manual. São Paulo: Vetor.
Snowling, M. J. (2004). Dislexia desenvolvimental: uma introdução e visão teórica geral. Em: M. Snowling \& J. Stackhouse. Dislexia, fala e linguagem. Porto Alegre: Artmed.

Suehiro, A. C. B. \& Santos, A. A. A. (2005). O Bender e as dificuldades de aprendizagem: um estudo de validade. Avaliação Psicológica, 4(1), 25-31.

Taylor, J. (2004). O desenvolvimento das habilidades de caligrafia. Em: M. Snowling \& J. Stackhouse. Dislexia, fala e linguagem. Porto Alegre: Artmed.

Van Hout, A. \& Estienne, F. (2001). Dislexias: descrição, avaliação, explicaşão, tratamento. Porto Alegre: Artmed.

Recebido em maio de 2006 Aprovado em abril de 2007

Sobre as autoras:

Acácia Aparecida Angeli dos Santos é doutora em Psicologia Escolar e Desenvolvimento Humano pela USP, docente da Graduação e do Programa de Pós-Graduação Stricto Sensu em Psicologia da Universidade São Francisco e bolsista produtividade do CNPq.

Lília Maíse de Jorge é mestre em Psicologia Escolar pela Pontifícia Universidade Católica de Campinas, doutoranda do Programa de Pós-Graduação Stricto Sensu em Psicologia da Universidade São Francisco e docente na Universidade de Taubaté. 
